\title{
Engineering vascular networks in porous polymer matrices
}

\author{
Martin C. Peters, ${ }^{1}$ Peter J. Polverini, ${ }^{2}$ David J. Mooney ${ }^{1,3,4}$ \\ ${ }^{1}$ Department of Biomedical Engineering, University of Michigan, 5213 Dental School, 1011 North University Avenue, \\ Ann Arbor, Michigan 48109-1078 \\ ${ }^{2}$ Dental School, University of Minnesota, Minneapolis, Minnesota 55455 \\ ${ }^{3}$ Department of Biologic \& Materials Sciences, University of Michigan, 5213 Dental School, 1011 North University \\ Avenue, Ann Arbor, Michigan 48109-1078 \\ ${ }^{4}$ Department of Chemical Engineering, University of Michigan, 5213 Dental School, 1011 North University Avenue, \\ Ann Arbor, Michigan 48109-1078
}

Received 9 March 2001; Revised 9 October 2001; accepted 2 November 2001

\begin{abstract}
Enhanced vascularization is critical to the treatment of ischemic tissues and the engineering of new tissues and organs. We have investigated whether sustained and localized delivery of vascular endothelial growth factor (VEGF) combined with transplantation of human microvascular endothelial cells (HMVECs) can be used to engineer new vascular networks. VEGF was incorporated and released in a sustained manner from porous poly(lactic-coglycolic acid) (PLG) matrices to promote angiogenesis at the transplantation site. VEGF could be incorporated and released in a biologically active form from PLG matrices, with the majority of VEGF release $(64 \%)$ occurring within 2 weeks. These matrices promoted a $260 \%$ increase in the density of host SCID mouse-derived capillaries invading the
\end{abstract}

matrices after 7 days of implantation, confirming the activity of the released VEGF. HMVECs were transplanted into SCID mice on PLG matrices, and organized to form immature human-derived vessels within 3 days. Functional vessels were observed within 7 days. Importantly, when HMVECs were transplanted on VEGF-releasing matrices, a $160 \%$ increase in the density of human-derived blood vessels was observed after 14 days. These findings suggest that combining elements of vasculogenesis and angiogenesis provides a viable and novel approach to enhancing local vascularization. (c) 2002 Wiley Periodicals, Inc. J Biomed Mater Res 60: 668-678, 2002

Key words: angiogenesis; drug delivery; endothelial cells; tissue engineering; VEGF

\section{INTRODUCTION}

There exists a critical need to promote angiogenesis in a variety of clinical situations, including the development of collateral vascularization in cases of tissue ischemia, wound healing, and in the emerging field of tissue engineering. Two potential routes exist to promote blood vessel development: (1) encourage new vessels to sprout from preexisting vessels in the native tissue with growth factors (angiogenesis), (2) transplant cells capable of differentiating into new vessels, in essence mimicking the process of vasculogenesis. In the nearly 30 years since Folkman ${ }^{1}$ first suggested a

Correspondence to: D. J. Mooney; e-mail: mooneyd@umich.edu Contract grant sponsor: National Institutes of Health; contract grant numbers: R29 DK50715, R01 DE13033

Contract grant sponsor: Curis Inc.

Contract grant sponsor: Whitaker Foundation (to M.C.P.)

(C) 2002 Wiley Periodicals, Inc. relationship between neovascularization and angiogenic molecules, tremendous progress has been made in understanding the process of angiogenesis. ${ }^{2}$ This understanding, coupled with the isolation and recombinant expression of angiogenic growth factors, has allowed many research groups to demonstrate the feasibility of using growth factors to enhance, or promote, the development of blood vessels. ${ }^{3,4}$ Therapeutic angiogenesis has already been used to successfully increase the capacity of collateral vascularization in cases of both peripheral ${ }^{5-7}$ and myocardial ischemia. ${ }^{8,9}$

Current studies of therapeutic angiogenesis deliver angiogenic growth factors through either a single, or repeated, injection directly into the circulation or target tissue to stimulate a response (e.g., migration, proliferation) from the cellular component of preexisting blood vessels. These approaches to delivery of growth factor risk promoting unwanted blood vessel formation (e.g., at the site of quiescent tumors) and toxic side effects. ${ }^{10-12}$ In addition, the bolus administration of growth factor cannot maintain a consistent concentra- 
tion at the desired site of angiogenesis, as demonstrated by vascular endothelial growth factor (VEGF), which has been shown to have an elimination half-life of less than $1 \mathrm{~h}$ following injection. ${ }^{8}$ Delivering angiogenic growth factors utilizing controlled drug delivery strategies, ${ }^{13,14}$ offers the potential to promote angiogenesis at a specific site while leaving the circulation free from high concentrations of growth factor.

A localized, sustained delivery strategy will broaden the applications of therapeutic angiogenesis to new fields, such as tissue engineering, in which developing vascular beds is a critical challenge. ${ }^{14}$ Tissue engineering seeks to bridge the gap between transplant organ supply and demand by engineering new functional tissues from relatively small cell populations. ${ }^{15}$ This general strategy to engineer tissues via cell transplantation utilizes cells that have been isolated from a patient and expanded in culture. One of the most significant challenges facing this approach is ensuring sufficient nutrient delivery to the transplanted cells. When cells are first transplanted into a host, on three-dimensional porous matrices, ${ }^{16}$ the area within the matrix is avascular and the transplanted cells are dependent on the diffusion of nutrients and waste for survival. This condition of limited transport is adequate if the tissue is small $(<1$-mm thick) or the cells' metabolic needs are low (e.g., chondrocytes). In the case of more metabolically demanding cell populations (e.g., hepatocytes), most cells die soon after transplantation ${ }^{17}$ because of mass transport limitations. ${ }^{18}$ Surrounding tissue from the host will naturally invade the porous matrix, ${ }^{19}$ bringing blood vessels, but this alone may be insufficient to provide necessary nutrients to cells within the matrix. By inducing the formation of additional blood vessels in these porous matrices, it may ultimately be possible to enhance the long-term survival of transplanted cells. ${ }^{14}$

Inductive means of engineering vascular networks may be limited if the ability of host blood vessels to respond to angiogenic molecules is compromised either by disease (e.g., impaired wound healing in diabetes) or prior medical treatments (e.g., radiation therapy). An alternative approach to neovascularization involves directly transplanting vessel-forming cells as a source for new blood vessels. ${ }^{20,21}$ Such vasculogenesis is normally observed only during development, ${ }^{22}$ but recent studies have demonstrated the feasibility of transplanting endothelial cells (ECs) ${ }^{21}$ or their precursors, ${ }^{23}$ to form neovessels. Implanting the cellular components of blood vessels may provide a strategy to improve neovascularization in certain situations because fewer ECs may be required to migrate from existing blood vessels.

This report addresses the possibility that localized delivery of growth factors may allow one to form new vascular beds in a localized region with a small total dose of the angiogenic molecule. In addition, the effect of locally delivered growth factors on blood vessel formation by transplanted ECs is also investigated. It is hypothesized that the released growth factors can act upon the transplanted ECs to promote survival, proliferation, and differentiation. We have developed and demonstrated a new in vivo system capable of sustained delivery of bioactive VEGF from a threedimensional cell transplantation matrix. The use of a highly porous polymer matrix enables one to define the area in which blood vessel development can be observed and create a potential space for EC transplantation. The controlled release of VEGF allowed the implanted matrices to directly control the behavior of the local cell populations and promoted the formation of blood vessels from both transplanted and native ECs.

\section{MATERIALS AND METHODS}

\section{Matrix formation and characterization}

A copolymer of D,L-lactide and glycolide (PLG) (85:15 molar ratio) (Boehringer Ingleheim, Germany) was used in a gas foaming/particulate leaching process ${ }^{24,25}$ to form matrices for VEGF and EC delivery. Granular PLG (5 mg; $106 \mu \mathrm{m}$ $<\mathrm{d}<250 \mu \mathrm{m}$ ) was mixed with $0.25 \mathrm{~mL}$ of an aqueous $0.1 \%$ alginate (ProNova, Oslo, Norway) solution, and $5 \mu \mathrm{g}$ of VEGF $_{165}$ (Intergen, Purchase, NY). The samples were lyophilized to form a powder, mixed with $100 \mathrm{mg}$ of $\mathrm{NaCl}$ (250 $\mu \mathrm{m}<\mathrm{d}<425 \mu \mathrm{m}$ ), compression molded into a disk (diameter $4.2 \mathrm{~mm}$, thickness $3 \mathrm{~mm}$ ), and foamed as previously described. ${ }^{24,25}$ Control matrices with $0,0.25,0.5,1$, and $2 \mathrm{~mL}$ of the $0.1 \%$ alginate solution, mentioned above, were also manufactured. The $\mathrm{NaCl}$ particles were leached from the matrices, and any alginate present was crosslinked, by immersing each matrix in $0.1 \mathrm{M} \mathrm{CaCl}_{2}$ for $24 \mathrm{~h}$. Matrices fabricated with this process using similar conditions have previously been documented to exhibit a porosity of $95 \%$, and an average pore size of $190 \mu \mathrm{m} .^{24}$

For scanning electron microscopy examination of the porous matrices, samples were dried and sputter-coated with gold. The permeability of the matrices' porous structure to cells was next examined by seeding matrices processed with $0,5,10,20$, and $40(\mathrm{w} / \mathrm{w}) \%$ alginate $(n=5)$ with $3 \times 10^{6}$ human (dermal) microvascular endothelial cells (HMVECs) (Cascade Biologics, Portland, OR) suspended in $50 \mu \mathrm{L}$ of a 1:1 mixture of medium 131 (Cascade Biologics) and growth factor-reduced Matrigel ${ }^{\circledR}$ (Becton Dickinson, Bedford, MA). Each matrix was allowed to absorb the cell suspension for 5 $\mathrm{min}$ in a $30-\mathrm{mm}$ Petri dish. The matrices were then removed from the dish and any cells remaining in the dish were counted in a ZM Coulter Counter (Coulter Electronics, Hialeah, FL) to ultimately determine the number of cells seeded into each matrix. The mechanical properties of the matrices $(n=10)$ were measured with an MTS compression tester (MTS, Minneapolis, MN) as previously described. ${ }^{24}$ 
In vitro release and characterization of VEGF

To determine the efficiency of incorporation and the kinetics of VEGF release, $0.3 \mu \mathrm{Ci}$ of ${ }^{125} \mathrm{I}$-labeled human recombinant $\mathrm{VEGF}_{165}(100 \mu \mathrm{Ci} / \mu \mathrm{g})$ (Biomedical Technologies Inc., Stoughton, MA) was added to samples $(n=5)$ as a tracer. ${ }^{26}$ Biological activity of the released VEGF was confirmed by placing VEGF-releasing matrices $(n=5)$, supported in transwells (12-mm, 3- $\mu \mathrm{m}$ pore diameter) (Corning, Cambridge, MA) into the media, directly over HMVECs actively growing in cell culture wells of a 12-well plate (10,000 cells seeded/well) (Corning). After $72 \mathrm{~h}$, before confluence was observed, the cultured cells were trypsinized to remove them from the dish and counted with a Coulter counter. The matrices in transwells were then moved to wells freshly seeded with 10,000 HMVECs and allowed to incubate for an additional $72 \mathrm{~h}$. The magnitude of growth stimulation was compared with control conditions in which cells were cultured in the presence of matrices containing no VEGF and with known concentrations of VEGF.

In vivo studies

Matrices $(n=4)$ were implanted in subcutaneous pockets (two per animal) on the dorsal region of 32, 7-9-week-old male SCID mice (cb17/SCID) (Taconic Labs, Germantown, NY). Matrices were sterilized in all experiments by immersion in $100 \%$ ethanol followed by a series of rinses $(4 \times 15$ min each) in phosphate buffered saline. All NIH guidelines for the care and use of laboratory animals (NIH Publication no. 85-23 Rev. 1985) were observed. Animals were anesthetized with an intramuscular injection of ketamine (87 mg/ $\mathrm{mL})$ and xylazine $(2.6 \mathrm{mg} / \mathrm{mL})$. For conditions including transplanted cells, immediately before matrix implantation, $3 \times 10^{6}$ HMVECs were suspended in $50 \mu \mathrm{L}$ of a 1:1 mixture of medium 131 and growth factor-reduced Matrigel, and allowed to absorb into the polymer matrix $5 \mathrm{~min}$. Matrices were retrieved after $3,5,7$, and 14 days, embedded in paraffin, and sectioned for histologic examination. Sections were either stained with Gill 3 hematoxylin (Sigma, St. Louis, MO) and eosin (Sigma), or immunostained with antibodies raised against human CD34 (QBEnd 10, diluted 1: 10) (Lab Vision, Fremont, CA), human CD31 (JC/70A, diluted 1:10) (Dayko, Carpinteria, CA), and human von Willebrand factor (polyclonal, diluted 1:2000) (Dayko) using a standard protocol. ${ }^{21}$ Briefly, sections were deparaffinized and washed for $5 \mathrm{~min}$ in $3 \% \mathrm{H}_{2} \mathrm{O}_{2}$ (Sigma) to quench endogenous peroxidases. The sections were then incubated for 10 min with terminator blocking solution (Biocare Medical, Walnut Creek, CA) to prevent nonspecific antibody-antigen interactions, and then allowed to incubate with the primary antibody for $1.5 \mathrm{~h}$ at $37^{\circ} \mathrm{C}$. For a secondary antibody, broadspectrum biotinylated universal link (Biocare Medical) was used for 20 min followed by a 15-min incubation with streptavidin-HRP (Biocare Medical). The HRP reaction was completed with 3,3'-diaminobenzidine (Zymed, South San Francisco, CA) for $30 \mathrm{~s}$. The sections were then visualized at $200 \times$, $400 \times$, and 1000x with an E-800 light microscope (Nikon). Four samples from each condition, with eight sections from each sample, were analyzed with NIH Image software (developed by the US National Institutes of Health).

\section{RESULTS}

\section{VEGF delivery system}

A novel model system was next developed for the sustained delivery of VEGF. We previously demonstrated that VEGF can be incorporated and released from polymer matrices ${ }^{25}$ but the processing yielded a poor efficiency of incorporation. A polysaccharide, alginate, was included in the matrices in these studies, in an effort to improve VEGF incorporation. Matrices were first examined to determine the effects of the alginate component on the matrices' porous structure for fear it could be negatively affected. Scanning electron micrographs of the PLG matrices with $5(\mathrm{w} / \mathrm{w}) \%$ alginate showed an open porous polymer matrix indistinguishable from PLG matrices processed without the addition of alginate [Fig. 1(A,B)] whereas the inclusion of 10,20 , or $40(\mathrm{w} / \mathrm{w}) \%$ alginate was observed to occlude the porous structure of the matrices [Fig. 1(C-E)]. In addition, when HMVECs were seeded onto matrices processed with various weight fractions of alginate, the permeability of the matrices to cells was observed to decrease as the alginate content was increased [Fig. 1(F)]. The difference in cell seeding measured between the $5(\mathrm{w} / \mathrm{w}) \%$ alginate conditions and those without alginate were not statistically significant. Matrices with 10, 20, and $40(\mathrm{w} / \mathrm{w}) \%$ alginate were omitted from other experiments because of their negative effects on the overall porous structure.

Inclusion of $5(\mathrm{w} / \mathrm{w}) \%$ of alginate into the matrices led to an overall VEGF incorporation efficiency of $74 \pm$ $3 \%$ whereas matrices lacking alginate demonstrated a final incorporation of only $27 \pm 3 \%$. The elastic modulus for $5(\mathrm{w} / \mathrm{w}) \%$ alginate-containing matrices was $287 \pm 84 \mathrm{kPa}$, whereas matrices without alginate measured $182 \pm 62 \mathrm{kPa}$, which is comparable to previous reports ${ }^{24,25}$ and sufficient to resist compression in vivo. When the data sets were compared, no statistically significant difference $(p>0.05)$ was noted between the two conditions. This result suggests that the inclusion of $5(\mathrm{w} / \mathrm{w}) \%$ alginate had no significant effect on the mechanical strength or structure of these matrices.

The ability of matrices to release the incorporated VEGF in a sustained manner was next assessed in vitro. The in vitro release profile of VEGF from all matrix processing conditions demonstrated similar controlled kinetics, with $40 \%$ of the VEGF released in the first $24 \mathrm{~h}$, an additional 10\% being released in the second $24 \mathrm{~h}$, and $2 \%$ /day being released for the next 7 days [Fig. 2(A)]. After day 9, at which time $60 \%$ of the VEGF had left the matrix, the release decreased to 

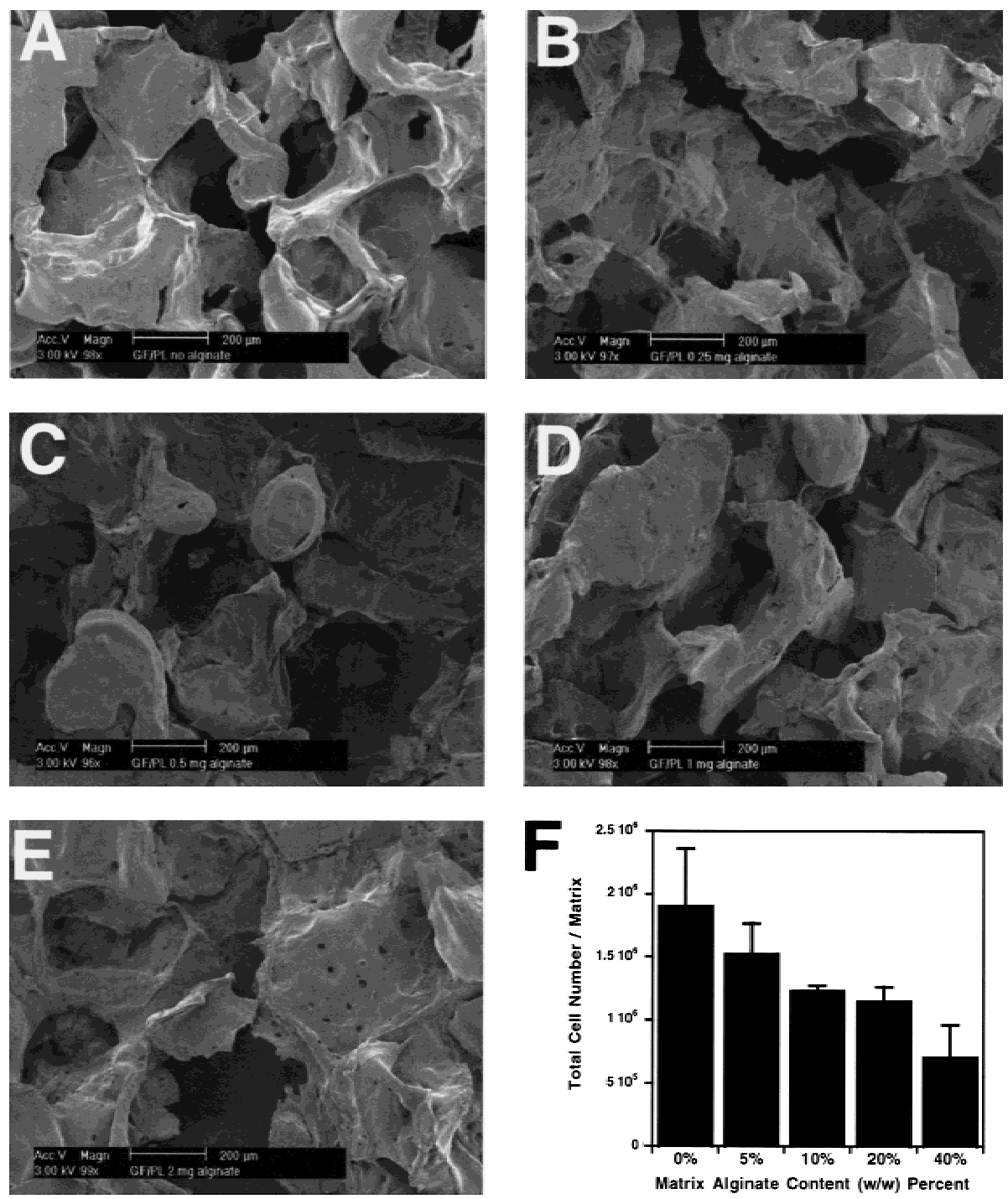

Figure 1. Scanning electron micrographs of PLG matrices processed with no alginate component (A), $5(\mathrm{w} / \mathrm{w}) \%$ alginate (B), $10(\mathrm{w} / \mathrm{w}) \%$ alginate $(\mathrm{C}), 20(\mathrm{w} / \mathrm{w}) \%$ alginate $(\mathrm{D})$, and $40(\mathrm{w} / \mathrm{w}) \%$ alginate $(\mathrm{E})$. When $3 \times 10^{6}$ HMVECs were seeded onto matrices $(n=5)$ with varying weight fractions of alginate, significantly fewer cells were observed to become settled onto the matrices as the fraction of alginate was increased (F). The differences between the number of cells retained in $0 \%$ and $5 \%$ alginate-containing matrices are not statistically significant.

$1 \% /$ day and continued for the remainder of the experiment. This rapid release of VEGF satisfies our goal of releasing the majority of the incorporated growth factor within 2 weeks, because this covers the time period in which the majority of transplanted cells die before they become integrated with the host tissue. ${ }^{27}$ A critical issue for any delivery vehicle is whether the growth factor has been denatured, or otherwise inactivated, during incorporation or storage in the polymer. ${ }^{28}$ The preservation of the VEGF's biological activity after incorporation and release was confirmed with an in vitro cell-based assay. VEGF-releasing matrices processed with $5(\mathrm{w} / \mathrm{w}) \%$ alginate and $5 \mu \mathrm{g}$ VEGF were allowed to release directly into tissue culture media in the presence of HMVECs. The released VEGF induced a statistically significant increase in cell proliferation in all times tested throughout 15 days of release [Fig. 2(B)]. The released VEGF was $>80 \%$ active as compared with control VEGF, even at the last time point tested.

To measure the physiological response induced by the sustained and localized delivery of VEGF, matrices processed with VEGF, and controls, were implanted subcutaneously into SCID mice and retrieved after 7 and 14 days (Fig. 3). Histological sections from the retrieved implants were stained with hematoxylin and eosin to visualize the blood vessels present within the matrices [Fig. 4(A-D)]. Blood vessel density was measured in the matrices and found to be approximately $260 \%$ greater in VEGF-releasing matrices, compared with control samples, after 7 days, and $70 \%$ greater after 14 days [Fig. 4(E)]. 

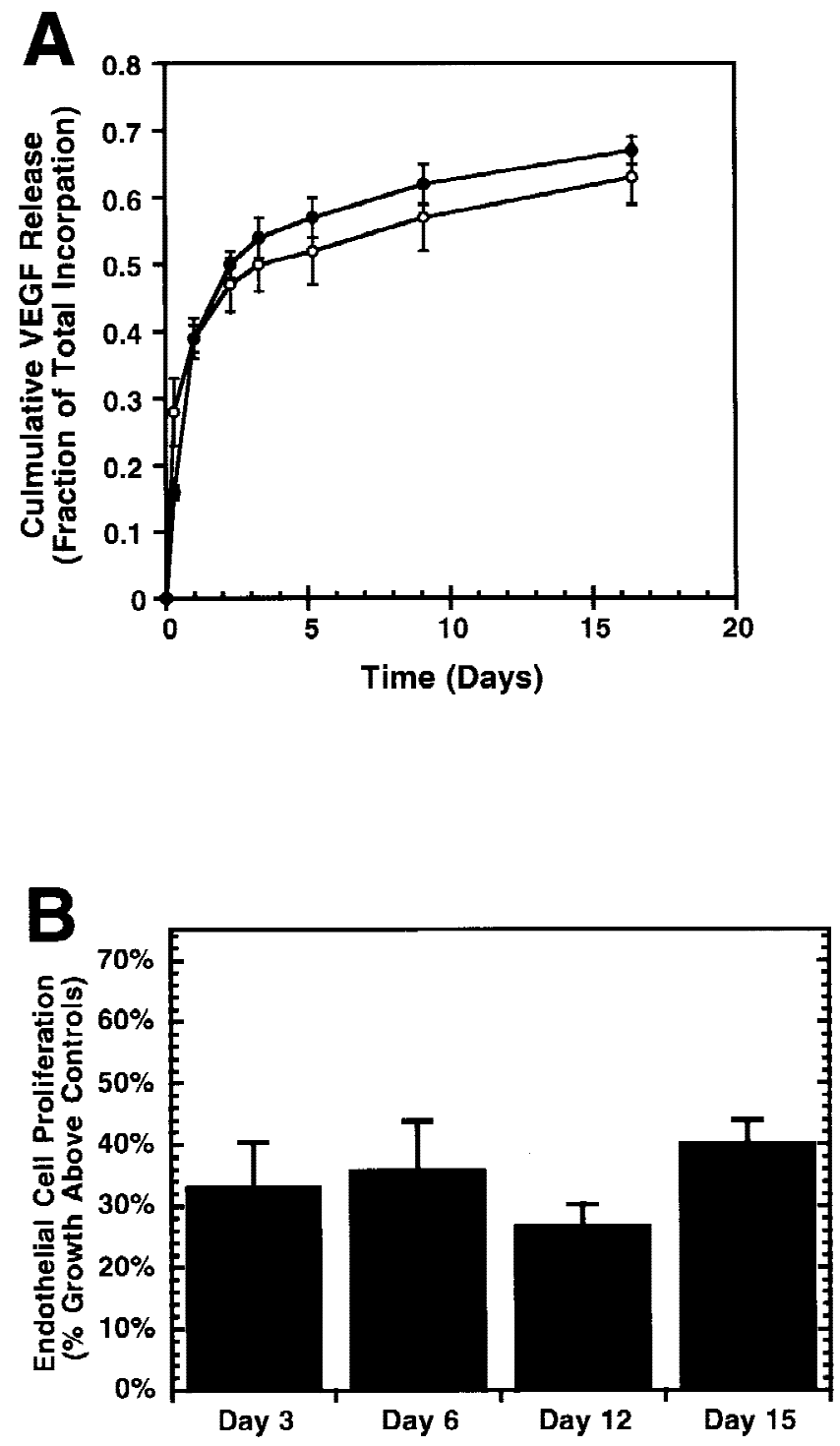

Figure 2. (A) Cumulative VEGF release in vitro $(n=5)$ from $5(\mathrm{w} / \mathrm{w}) \%$ alginate-containing matrices $(\bullet)$ and matrices processed without alginate $(O)$. Values represent mean and standard deviation. (B) Biological activity of VEGF released from $5(\mathrm{w} / \mathrm{w}) \%$ alginate-containing matrices $(n=6)$. Culture wells containing VEGF-releasing matrices showed a statistically significant $(p<0.05)$ increase in cell proliferation when compared with the growth observed in control samples. Values represent the fractional increase in cell growth over and above the growth observed in control samples.

\section{Combined VEGF delivery and EC transplantation}

HMVECs were transplanted into SCID mice on matrices to confirm our ability to engineer functional new blood vessels from transplanted cells. Sections from tissues removed at various times were stained for the presence of CD34, CD31, and von Willebrand factor. Positive CD34 and CD31 staining was specific to transplanted HMVECs, and found only within the space defined by the polymer matrices. Positive von Will-
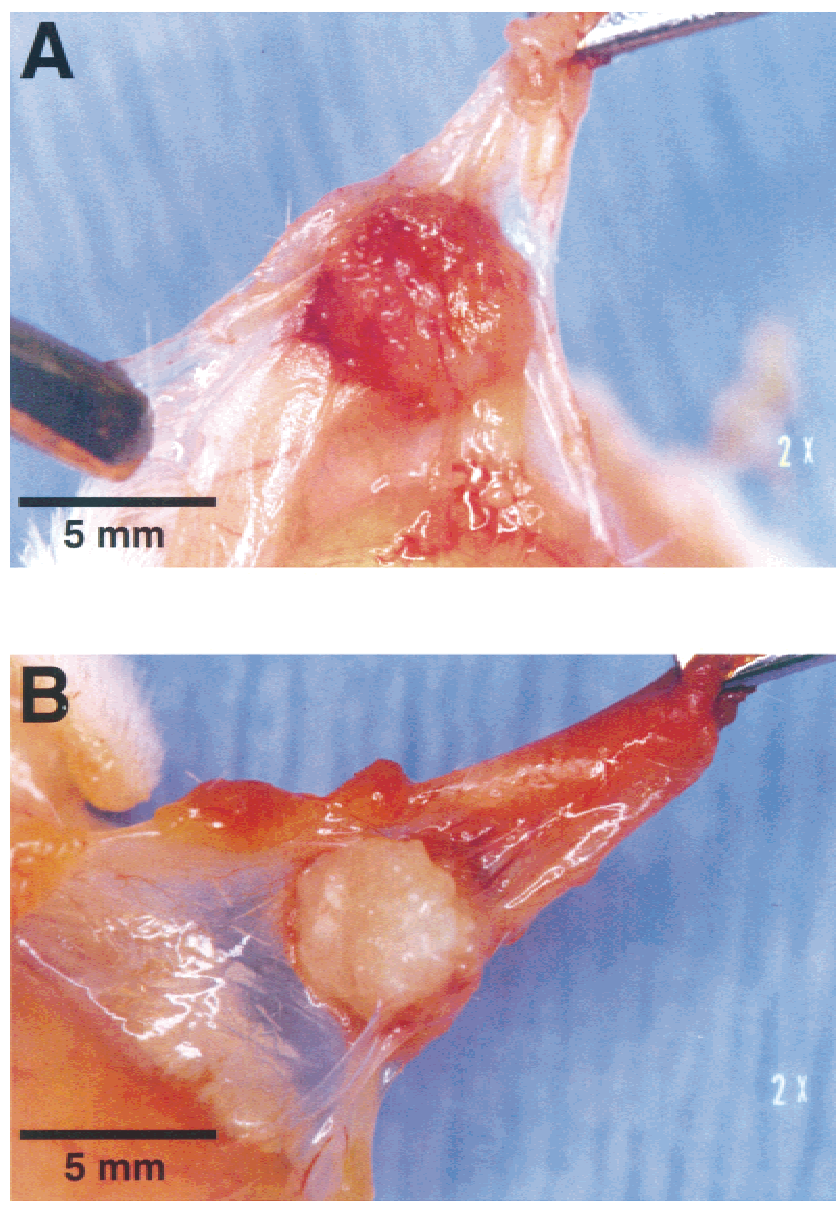

Figure 3. Gross photomicrographs of typical VEGFreleasing (A) and control (B) PLG matrices, 14 days after implantation. [Color figure can be viewed in the online issue, which is available at www.interscience.wiley.com.]

ebrand factor staining was observed only in large mouse-derived blood vessels outside the tissue actively invading the matrices (not shown). The CD34 positively stained HMVECs clearly illustrate the time course of blood vessel development from transplanted ECs. Transplanted HMVECs are initially fully rounded, but after 3 days, the cells within $100 \mu \mathrm{m}$ of the matrix boundary have begun to differentiate into loosely defined large rounded structures [Fig. 5(A,B)]. After 5 days, these structures have compartmentalized to form neovessels along the edge of mouse tissue, which is invading the matrix, but no evidence of vessel function can be found [Fig. 5(C)]. Mature humanderived vessels carrying mouse blood are observed after 7 and 14 days [Fig. 5(D,E)]. The pattern of CD31 staining (not shown) was indistinguishable from the results obtained with CD34.

Finally, to determine whether it is possible to modulate the ability of transplanted HMVECs to form vascular networks, HMVECs were transplanted into SCID mice on matrices releasing biologically active VEGF. Histological sections stained with anti-human 

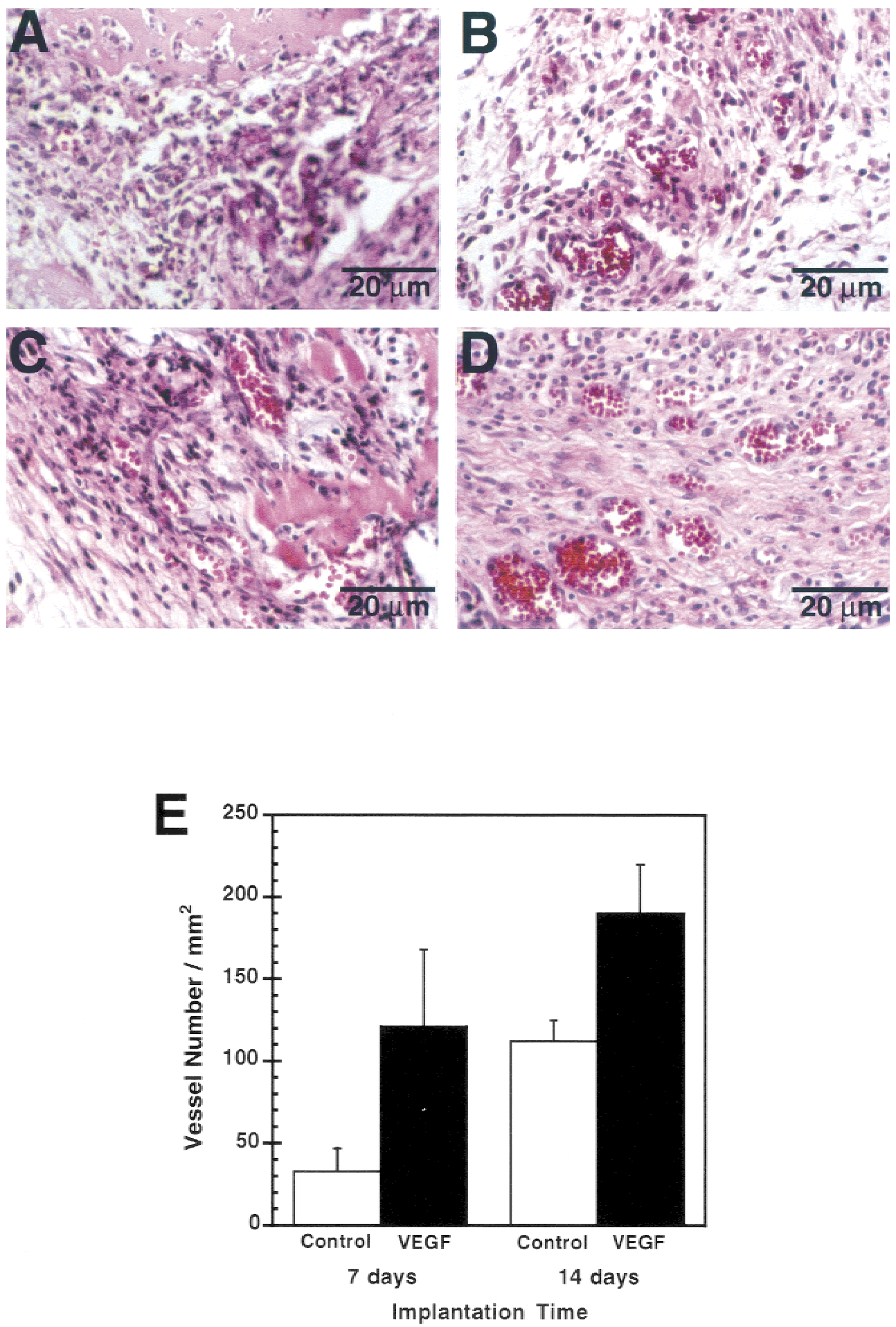

Figure 4. In vivo response to VEGF-releasing matrices $(n=4)$. Matrices were retrieved after $1(\mathrm{~A}$ and $\mathrm{B})$ and $2(\mathrm{C}$ and $\mathrm{D})$ weeks. Matrices releasing VEGF (B and D), and control samples (A and C), were photographed at original magnification 400x. (E) The density of blood vessels, measured in each implant by manual counting at 200× magnification, was significantly greater $(p<0.05)$ in VEGF-releasing matrices, compared with controls, at both time points. [Color figure can be viewed in the online issue, which is available at www.interscience.wiley.com.]

CD34 antibodies were again used to measure the density of human-derived vessels in the matrices. No statistically significant difference between the control (HMVECs, no VEGF) and experimental condition
(HMVECs and VEGF) was noted at 7 days. However, after 14 days, the VEGF-releasing condition showed a $160 \%$ increase in human-derived vessel density, compared with controls (Fig. 6). The lack of an effect at the 

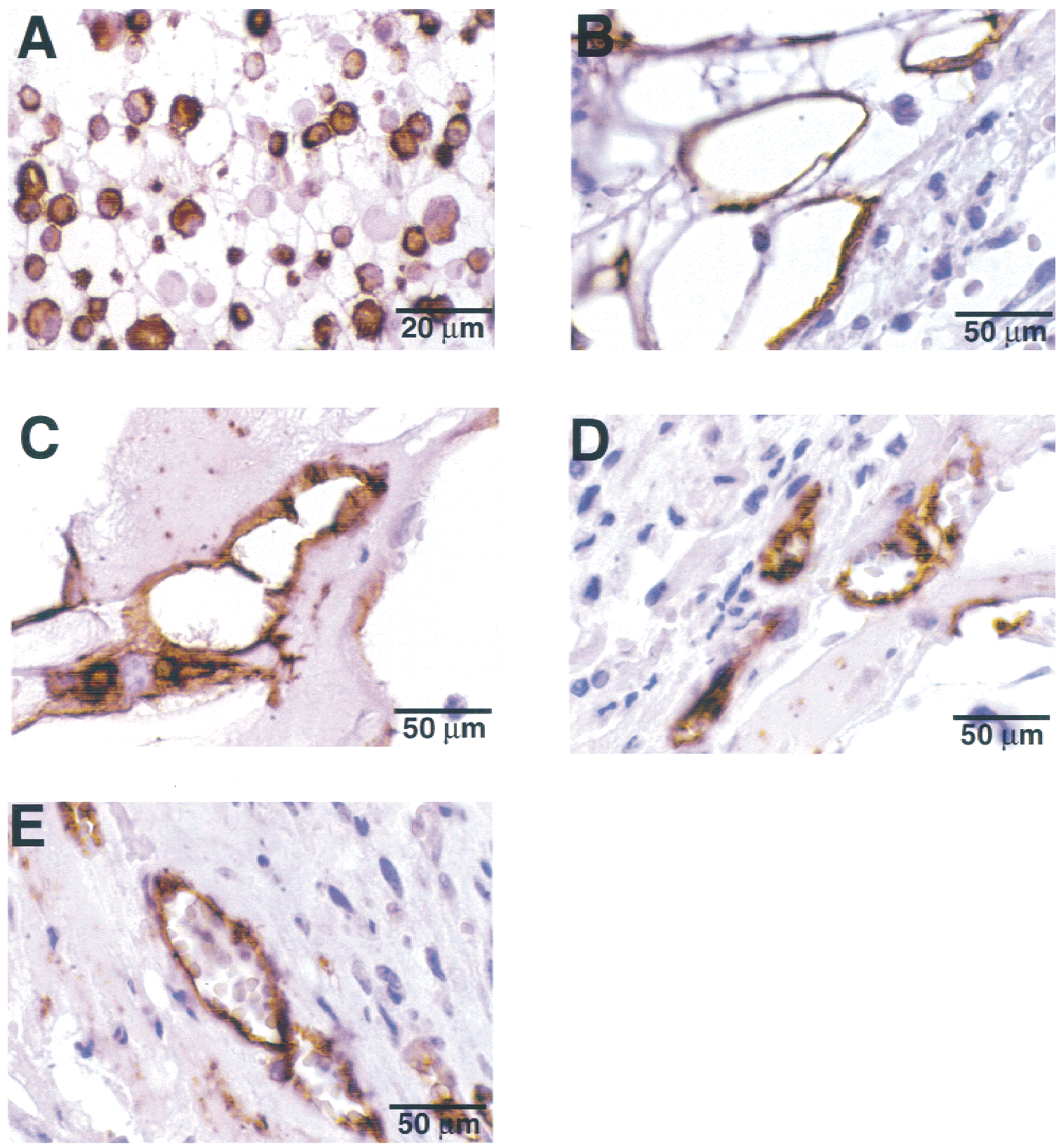

Figure 5. Photomicrographs of blood vessels developing from transplanted HMVECs. (A) Matrix seeded with HMVECs, and stained for human CD34 before implantation (original magnification $\times 400$ ). Implanted matrices, with HMVECs retrieved after 3 (B), 5 (C), 7 (D), and 14 days (E), stained for human CD34, and photographed at original magnification $\times 1000$.Vessels in (D) and (E) are functional as indicated by the presence of circulating red blood cells. [Color figure can be viewed in the online issue, which is available at www.interscience.wiley.com.]

early time points likely reflects an inability of the transplanted HMVECs to respond to the VEGF until organized to some extent. The number of host-derived blood vessels in the matrices was also quantified, and sustained delivery of VEGF again led to increases in the density of mouse-derived blood vessels at both 7 and 14 days. The magnitude of the mouse-derived blood vessel density increases were similar to that noted with VEGF delivery alone [Fig. 4(E)].

\section{DISCUSSION}

VEGF delivery and EC transplantation are promising approaches to promote therapeutic angiogenesis for a number of clinical applications. ${ }^{29}$ We have developed a model system that allows for combined VEGF delivery and EC transplantation. This system demonstrates that sustained release of VEGF from polymer matrices can increase the overall density of 

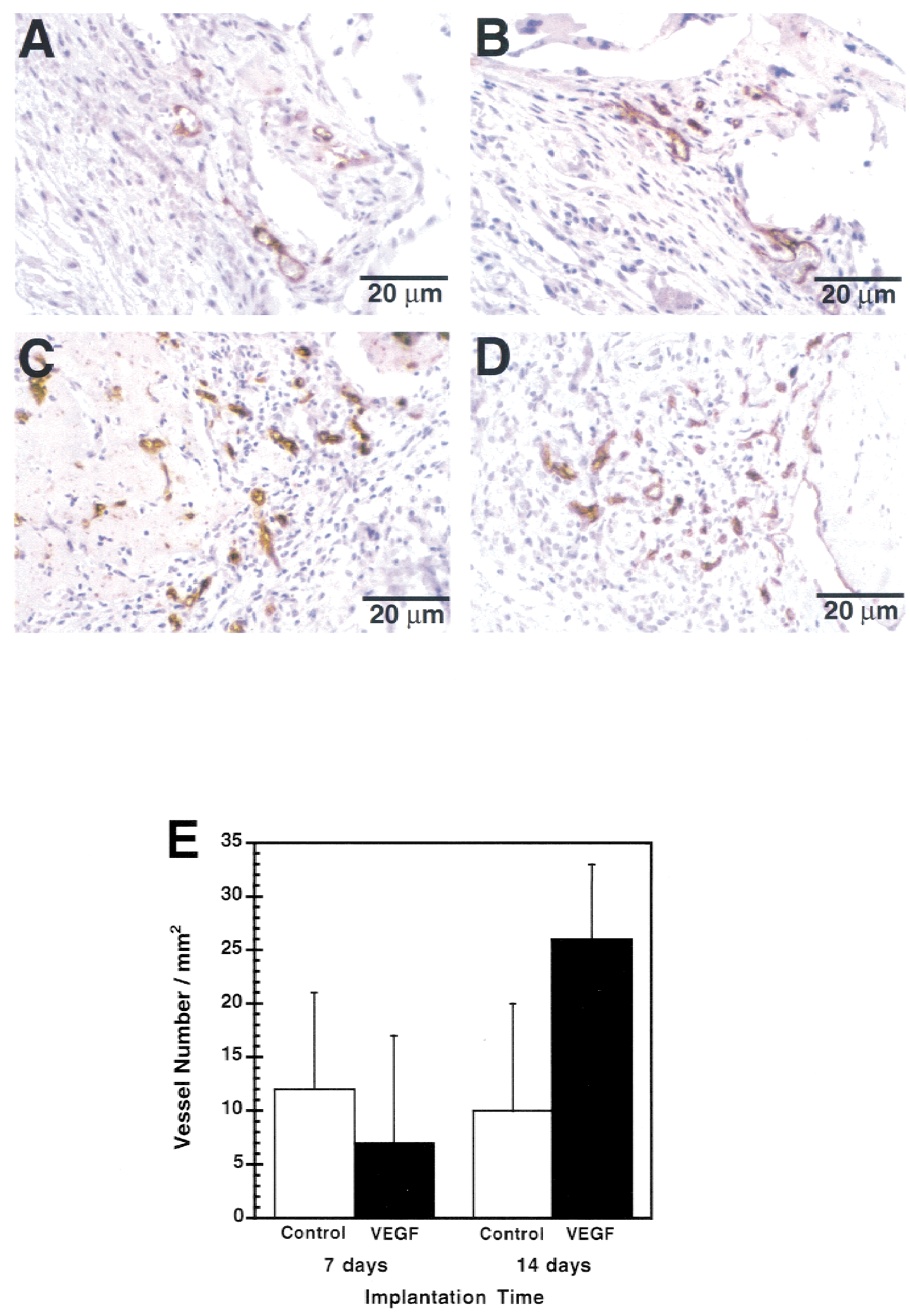

Figure 6. Human-derived blood vessel response to VEGF-releasing matrices. Matrices $(n=4 /$ time point/condition) were retrieved after 1 and 2 weeks. The density of HMVECs containing blood vessels in VEGF-releasing matrices increased significantly $(p<0.05)$ at 14 days in comparison with no-VEGF matrices. No statistically significant difference exists between the two conditions at the 7-day time point. [Color figure can be viewed in the online issue, which is available at www. interscience.wiley.com.] 
blood vessels present in the tissue growing in and around the matrix. In addition, this system demonstrates that providing a local source for VEGF increases the ability of transplanted HMVEC to integrate into the host vasculature and form functional blood vessels. These results suggest it may be possible to use a polymer-based delivery system to engineer more extensive vascular networks and collateral vascularization, or to create better environments for transplanted cell survival and proliferation.

Using a localized sustained release system for growth factors may greatly enhance the desired formation of local blood vessels, while eliminating the risk of toxic side effects ${ }^{12}$ and unwanted blood vessels developing at other sites in the body. Localized delivery as opposed to systemic delivery limits the VEGF's area of effect because large masses of VEGF do not enter the circulation. Sustained release at a local site confers additional advantages to our VEGF delivery system by further reducing the mass of VEGF needed to promote the desired effects. In contrast, a continuous introduction of new growth factor is used in an effort to maintain a stable concentration in most previously published work. ${ }^{30,31}$ Free VEGF is rapidly taken up by cells and degraded, or bound up by extracellular matrix molecules, ${ }^{5}$ which quickly decreases the concentration of injected VEGF. For example, repeated intravenous injections of $45 \mu \mathrm{g}$ of $\mathrm{VEGF}^{31}$ have been shown to induce no significant physiological effect, whereas we have demonstrated that the controlled release of only $3.75 \mu \mathrm{g}$ of VEGF is able to promote a measurable and continuous increase in blood vessel density for at least 2 weeks after implantation. This increase in vascularization was noted within and immediately adjacent to the scaffold. The dose of delivered VEGF was not varied in the current study. However, we anticipate that as the delivered dose is increased, the distance from the matrix within the surrounding tissue that exhibited an increased blood vessel density would rise in parallel. The magnitude of the observed blood vessel density increase in this study is comparable to other studies using systemic delivery of up to $1 \mathrm{mg}$ of VEGF. ${ }^{32,33}$

VEGF can be released in a controlled manner, from the matrices, in an intact and biologically active form. These newly developed matrices are physically indistinguishable from the gas-foamed matrices previously described.$^{24}$ In addition, they demonstrate improved VEGF incorporation and release kinetics compared with previously developed VEGF-delivery matrices. ${ }^{25}$ It is possible that the inclusion of alginate in the PLG matrices may alter the VEGF presentation to the target cells (e.g., released VEGF may be complexed with alginate chains). However, the release kinetics of VEGF from scaffolds was similar with or without alginate (Fig. 2), and the bioactivity of VEGF released from alginate-containing matrices was similar to that previ- ously measured when VEGF was released from alginate-free matrices. ${ }^{25}$ Our goal in this study was for the VEGF to be released relatively rapidly. The release of the incorporated VEGF is observed to occur in vitro as a large initial burst followed by a slower sustained release of the remaining factor. The initial release is likely due to the dissolution of lyophilized VEGF absorbed to the surface of the PLG, or release of VEGF contained within surface-accessible pores. The subsequent slower release rate is likely controlled by degradation of the PLG and diffusion of the VEGF through the matrix. In any case, it is probable that the significant burst observed in vitro is attenuated in vivo because of the absence of buffer solution washing completely through the porous matrix. In addition, the matrices are surrounded by wounded tissue after implantation, where plasma proteins freely adsorb onto the matrix and fibrovascular tissue rapidly grows into the porous openings along the matrix surface. These environmental changes will likely alter the degradation rate of the polymer matrix and limit the diffusion distance of the released VEGF.

HMVECs transplanted on PLG matrices were able to form new blood vessels. The transplanted cells rapidly joined with the host vasculature to form hybrid vessels without outside stimulus, but when this same experiment was repeated with a VEGF-releasing matrix, the result was a significant increase in the number of blood vessels derived from transplanted EC within 14 days. In this case, the effect of the VEGF is likely two-fold. It may contribute both to the survival of the transplanted cells via the activation of apoptosis suppression genes, ${ }^{34,35}$ and encourage EC differentiation into neovessels. It is important to note that the delivery of VEGF alone led to a dramatic increase in hostderived blood vessels invading the matrix space, and the density of host-derived blood vessels was approximately one order of magnitude higher than the density of blood vessels formed by transplanted ECs. However, in situations in which the host tissue has a compromised ability to respond to angiogenic factors, it may be necessary to provide ECs within the scaffold to enhance neovascularization. In addition, transplantation of genetically modified ECs may be useful as an approach to deliver macromolecular drugs to the systemic circulation. In either of these situations, it will clearly be desirable to maximize the integration of the transplanted ECs with the host vasculature.

Drug-releasing matrices capable of cell transplantation represent a potentially powerful new model of vascularization, with the ability to control which cell types are presented at a site and the local concentration of both promoters and inhibitors of angiogenesis. ${ }^{25,36}$ By virtue of the matrices' spatial and temporal control over drug release, greater insight might be gained into the complex and dynamic process of angiogenesis and vasculogenesis for cancer research as 
well as tissue engineering applications. When compared with other methods of controlled drug delivery (e.g., microspheres), drug-releasing scaffolds have several advantages. Porous PLG matrices create and define a space for study, and have been shown to provide an effective support for the growth of a variety of transplanted cells and tissues, including tumors. ${ }^{36}$ This grants researchers the ability to easily incorporate any cell type, or even genetically modified cells, into the experimental system. Blood vessel development can be followed in either transplanted ECs or precursors, and ECs originating from host blood vessels. ${ }^{21}$ These drug-releasing matrices will become even more valuable as investigators continue to identify and implicate new molecules in the angiogenic cascade. Recently, attention has focused on the temporal importance of growth factor presentation in angiogenesis. ${ }^{37}$ Drug-releasing polymer matrices also offer the potential to release a combination of growth factors from a single matrix. These systems may broadly aid in understanding the biochemical pathways that depend on a number of simultaneously or sequentially delivered soluble signals.

\section{References}

1. Folkman J. Tumor angiogenesis: Therapeutic implications. N Engl J Med 1971;285:1182-1186.

2. Folkman J. Angiogenesis and angiogenesis inhibition: An overview. EXS 1997;79:1-8.

3. Isner JM, Asahara T. Angiogenesis and vasculogenesis as therapeutic strategies for postnatal neovascularization. J Clin Invest 1999;103:1231-1236.

4. Henry TD. Therapeutic angiogenesis. Br Med J 1999;318:15361539.

5. Takeshita S, Zheng LP, Brogi E, Kearney M, Pu LQ, Bunting S, Ferrara N, Symes JF, Isner JM. Therapeutic angiogenesis: A single intraarterial bolus of vascular endothelial growth factor augments revascularization in a rabbit ischemic hind limb model. J Clin Invest 1994;93:662-670.

6. Baumgartner I, Isner JM. Stimulation of peripheral angiogenesis by vascular endothelial growth factor (VEGF). Vasa 1998; 27:201-206.

7. Lee KY, Peters MC, Anderson KW, Mooney DJ. Controlled growth factor release from synthetic extracellular matrices. Nature 2000;408:998-1000.

8. Lazarous DF, Shou M, Scheinowitz M, Hodge E, Thirumurti V, Kitsiou AN, Stiber JA, Lobo AD, Hunsberger S, Guetta E, Epstein SE, Unger EF. Comparative effects of basic fibroblast growth factor and vascular endothelial growth factor on coronary collateral development and the arterial response to injury. Circulation 1996;94:1074-1082.

9. Hendel RC, Henry TD, Rocha-Singh K, Isner JM, Kereiakes DJ, Giordano FJ, Simons M, Bonow RO. Effect of intracoronary recombinant human vascular endothelial growth factor on myocardial perfusion: Evidence for a dose-dependent effect. Circulation 2000;101:118-121.

10. Mazue G, Bertolero F, Jacob C, Sarmientos P, Roncucci R. Preclinical and clinical studies with recombinant human basic fibroblast growth factor. Ann NY Acad Sci 1991;638:329-340.
11. Mazue G, Bertolero F, Garofano L, Brughera M, Carminati P. Experience with the preclinical assessment of basic fibroblast growth factor (bFGF). Toxicol Lett 1992;64-65 Spec No: 329338.

12. Mazue G, Newman AJ, Scampini G, Della Torre P, Hard GC, Iatropoulos MJ, Williams GM, Bagnasco SM. The histopathology of kidney changes in rats and monkeys following intravenous administration of massive doses of FCE 26184, human basic fibroblast growth factor. Toxicol Pathol 1993;21:490-501.

13. Langer R. Drug delivery and targeting. Nature 1998;392:5-10.

14. Peters MC, Mooney DJ. Growth factor delivery from tissue engineering matrices: Inducing angiogenesis to enhance transplanted cell engraftment. In: Park K, Mrsny RJ, editors. Controlling drug delivery: Designing technologies for the future. Washington, DC: American Chemical Society; 2000. p 157-167.

15. Langer R, Vacanti JP. Tissue engineering. Science 1993;260:920926.

16. Putnam AJ, Mooney DJ. Tissue engineering using synthetic extracellular matrices. Nat Med 1996;2:824-826.

17. Mooney DJ, Sano K, Kaufmann PM, Majahod K, Schloo B, Vacanti JP, Langer R. Long-term engraftment of hepatocytes transplanted on biodegradable polymer sponges. J Biomed Mater Res 1997;37:413-420.

18. Colton CK. Implantable biohybrid artificial organs. Cell Transplant 1995;4:415-436.

19. Wake MC, Patrick CW Jr, Mikos AG. Pore morphology effects on the fibrovascular tissue growth in porous polymer substrates. Cell Transplant 1994;3:339-343.

20. Holder WD, Gruber HE, Roland WE, Moore AL, Culberson $\mathrm{CR}$, Loebsack AB, Burg KL, Mooney DJ. Increased vascularization and heterogeneity of vascular structures occurring in polyglycolide matrices containing aortic endothelial cells implanted in the rat. Tissue Eng 1997;3:149-160.

21. Nor JE, Peters MC, Christensen J, Sutorik MM, Mooney DJ, Polverini PJ. Engineering and characterization of functional human microvessels in immunodeficient mice. Lab Invest 2001;81:453-463.

22. Polverini PJ. The pathophysiology of angiogenesis. Crit Rev Oral Biol Med 1996;6:230-247.

23. Takahashi T, Kalka C, Masuda H, Chen D, Silver M, Kearney M, Magner M, Isner JM, Asahara T. Ischemia- and cytokineinduced mobilization of bone marrow-derived endothelial progenitor cells for neovascularization. Nat Med 1999;5:434-438.

24. Harris LD, Kim BS, Mooney DJ. Open pore biodegradable matrices formed with gas foaming. J Biomed Mater Res 1998;42: 396-402.

25. Sheridan MH, Shea LD, Peters MC, Mooney DJ. Bioabsorbable polymer scaffolds for tissue engineering capable of sustained growth factor delivery. J Controlled Release 2000;64:91-102.

26. Peters MC, Isenberg BC, Rowley JA, Mooney DJ. Release from alginate enhances the biological activity of vascular endothelial growth factor. J Biomater Sci Polym Ed 1998;9:1267-1278.

27. Mooney DJ, Kaufmann PM, Sano K, Schwendeman SP, Majahod K, Schloo B, Vacanti JP, Langer R. Localized delivery of epidermal growth factor improves the survival of transplanted hepatocytes. Biotechnol Bioeng 1996;50:422-429.

28. Cleland JL, Powell MF, Shire SJ. The development of stable protein formulations: A close look at protein aggregation, deamidation, and oxidation [published erratum appears in Crit Rev Ther Drug Carrier Syst 1994;11(1):60]. Crit Rev Ther Drug Carrier Syst 1993;10:307-377.

29. Isner JM. Angiogenesis: a "breakthrough" technology in cardiovascular medicine. J Invasive Cardiol 2000;12(Suppl A): 14A-17A.

30. Baffour R, Berman J, Garb JL, Rhee SW, Kaufman J, Friedmann $P$. Enhanced angiogenesis and growth of collaterals by in vivo 
administration of recombinant basic fibroblast growth factor in a rabbit model of acute lower limb ischemia: Dose-response effect of basic fibroblast growth factor. J Vasc Surg 1992;16:181191.

31. Banai S, Jaklitsch MT, Shou M, Lazarous DF, Scheinowitz M, Biro S, Epstein SE, Unger EF. Angiogenic-induced enhancement of collateral blood flow to ischemic myocardium by vascular endothelial growth factor in dogs. Circulation 1994;89: 2183-2189.

32. Bauters C, Asahara T, Zheng LP, Takeshita S, Bunting S, Ferrara N, Symes JF, Isner JM. Site-specific therapeutic angiogenesis after systemic administration of vascular endothelial growth factor. J Vasc Surg 1995;21:314-325.

33. Takeshita S, Rossow ST, Kearney M, Zheng LP, Bauters C, Bunting S, Ferrara N, Symes JF, Isner JM. Time course of increased cellular proliferation in collateral arteries after administration of vascular endothelial growth factor in a rabbit model of lower limb vascular insufficiency. Am J Pathol 1995; 147:1649-1660.

34. Nor JE, Christensen J, Mooney DJ, Polverini PJ. Vascular endothelial growth factor (VEGF)-mediated angiogenesis is associated with enhanced endothelial cell survival and induction of Bcl-2 expression. Am J Pathol 1999;154:375-384.

35. Nor JE, Mitra RS, Sutorik MM, Mooney DJ, Castle VP, Polverini PJ. Thrombospondin-1 induces endothelial cell apoptosis and inhibits angiogenesis by activating the caspase death pathway. J Vasc Res 2000;37:209-218.

36. Nor JE, Christensen J, Liu J, Peters M, Mooney DJ, Strieter RM, Polverini PJ. Upregulation of Bcl-2 in endothelial cells induces IL-8 expression and enhances tumor angiogenesis and tumor growth. Cancer Res 2001;61:2183-2188.

37. Darland DC, D'Amore PA. Blood vessel maturation: vascular development comes of age. J Clin Invest 1999;103:157-158. 